\title{
Effects of diacerein on cartilage and subchondral bone in early stages of osteoarthritis in a rabbit model
}

\author{
María Permuy ${ }^{1 *}$, David Guede ${ }^{2}$, Mónica López-Peña ${ }^{1}$, Fernando Muñoz ${ }^{1}$, Jose-Ramón Caeiro ${ }^{3}$ \\ and Antonio González-Cantalapiedra'
}

\begin{abstract}
Background: Osteoarthritis is thought to be the most prevalent chronic and disabling joint disease in animals and humans. At present, there is no ideal treatment option. The aim of this study was to assess the effects of the treatment with oral diacerein on articular cartilage, synovial membrane and subchondral bone in an experimental rabbit model of osteoarthritis by micro-CT evaluation and histological analysis. To this purpose, osteoarthritis was surgically induced on one knee of 16 rabbits using the contralateral knee as healthy controls. Treatment was started three weeks later and lasted eight weeks. Animals were divided into two groups for treatment: Placebo (treated daily with oral saline) and diacerein (treated orally with $1.5 \mathrm{mg} / \mathrm{kg} /$ day of diacerein).

Results: Sample analysis revealed that this model induced osteoarthritis in the operated knee joint. Osteoarthritis placebo group showed a significant increase in non-calcified cartilage thickness and volume with respect to the control placebo group and important changes in the synovial membrane; whereas the parameters measured in subchondral bone remained unchanged. In the osteoarthritis diacerein-treated group the results showed an improvement with respect to the OA placebo group in all parameters, although the results were not statistically significant.
\end{abstract}

Conclusion: The results of this animal study suggested that the diacerein treatment for OA may be able to ameliorate the swelling and surface alterations of the cartilage and exert an anti-inflammatory effect on the synovial membrane, which might contribute to OA improvement, as well as an anabolic effect on subchondral trabecular bone.

Keywords: Bone, Cartilage, Diacerein, Osteoarthritis, Rabbit model, Synovial membrane

\section{Background}

Osteoarthritis $(\mathrm{OA})$ is a heterogeneous chronic disease affecting all tissues of the synovial joint. It is now accepted that the disease etiology is multiple and involves various mechanical, biochemical and genetic factors, as well as molecular and enzymatic feedback loops. Osteoarthritic changes are primarily the result of a disturbance in the remodelling processes of tissues resulting from the failure of cells to maintain a homeostatic balance; as the disease advances, the catabolic process exceeds the anabolic ones leading to progressive joint tissue lesions.

\footnotetext{
* Correspondence: maria.permuy@usc.es

'Veterinary Clinical Sciences, University of Santiago de Compostela (USC),

Campus Universitario, s/n, 27002, Lugo, Spain

Full list of author information is available at the end of the article
}

The morphological changes observed in OA include a variable degree of synovial inflammation, principally at the clinical stage of the disease, which in turn produces inflammatory mediators (inflammatory cytokines), which play a pivotal role in the pathophysiological mechanisms of the OA $[1,2]$.

The pharmacological treatment of OA includes different types of drugs that can be classified on basis of their mode of action. Current treatment options are focused on pain relief and improvement of joint function, including analgesics and non-steroidal anti-inflammatory drugs; unfortunately, their use in chronic treatments has been limited by the deleterious side effects on cartilage [3] and gastrointestinal tract [4]. Recent studies have described drugs with the ability of providing symptomatic 
relief by targeting the underlying pathology of OA, particularly in cartilage and subchondral bone, and with less secondary effects; such agents have been classified as symptomatic slow-acting drugs for OA (SYSADOAs) which are expected to delay, stabilise or reverse the pathological changes in OA joints, limiting the progression of the disease [5]. This group of drugs includes diacerein, an anthraquinone derivative (4,5-diacetyloxy9,10-dioxo-anthracene-2-carboxylic acid), which is an oral anti-inflammatory, analgesic and antipyretic agent developed specifically for the treatment of OA.

It is actually well-known the contribution of proinflammatory cytokines to cartilage degradation in OA $[6,7]$ including interleukin-1 (IL-1) which stimulates the degradation process of cartilage and suppresses cartilage matrix synthesis. Rhein, the active metabolite of diacerein, has shown in vitro and in vivo the inhibition of the IL- $1 \beta$, as well as the reduction of the collagenase production by joint chondrocytes, the reduction of fibrinolytic activity in synovial fluid $[8,9]$ and the stimulation of the production of cartilage growth factors [10]. This way of action turned diacerein into an interesting option for the treatment of $\mathrm{OA}$ without the side effects of NSAIDs [11].

In a previous study in dogs [12], diacerein treatment significantly reduced the severity of morphologic changes in the knee joint compared to placebo, using the SFA (Societé Française d'Arthroscopie) score and, in the same study, the Mankin scores were lower for the diacerein group. In another study in rats [13], the treatment with diacerein produced the best functional results represented by a greater joint extension. In addition, several trials in humans suggested beneficial role of diacerein in hip and knee OA with results similar to those obtained by NSAIDs during the active treatment phase [14]. Bartels et al. (2010) [15] described a small reduction of pain in patients treated with diacerein during the first 6 months, whereas Pavelka et al. (2007) [16] reported a statistical superiority of diacerein versus placebo in pain control and tenderness of the joint; Singh et al. (2012) [17] reported an additive effect with diclofenac for pain and improvement of the joint motion.

The aim of this study was to test the effectiveness of the treatment with oral diacerein in an experimental early rabbit model of OA; histology and histomorphometry techniques were used and the results were compared with a new methodology to determine the cartilage and subchondral bone structure, using micro-computed tomography (micro-CT) without contrast techniques.

\section{Methods}

Pre- and postoperative care. Osteoarthritis induction Sixteen adult female (6-7 months old, mean weight 5 $\mathrm{Kg}$ ) healthy New Zealand white rabbits (Granja San
Bernardo, Navarra, Spain) were used, after approval of the protocol by the Ethical Committee of the University of Santiago de Compostela (Reference number: AE-LU002/09/FUN01/PAT.(06)E). All animals were subjected to surgery and throughout the procedures they were housed in cages at the Animal Research Facility, University of Santiago de Compostela, Lugo, Spain. The procedures were performed according to local and European regulations on care and use of research animals, and this paper was written in accordance with the ARRIVE statement [18]. The rabbits were monitored daily during the entire experimental procedure by an accredited veterinarian, trained in laboratory animal science.

The protocol used has been deeply described in a recently published article [19]. Summarising, OA was induced by anterior cruciate ligament transection (ACLT) and partial medial meniscectomy of one knee (randomly chosen) using the contralateral knee as untreated control. Animals were anaesthetised using isoflurane (2.5-4 \%, Isova-vet, Schering-Plough, Madrid, Spain) after premedication with a combination of medetomidine $(50 \mu \mathrm{g} /$ Kg IM; Domtor, Esteve, Barcelona, Spain) and ketamine (25 mg/Kg IM; Imalgène 1000, Merial, Toulouse, France). Each animal received peri- and post-operative buprenorphine (1 mg/Kg IM; Buprex, RB Pharmaceuticals, Berkshire, UK), antibiotic prophylaxis with enrofloxacin for a week $(15 \mathrm{mg} / \mathrm{Kg} \mathrm{SC}$ once a day; Ganadexil $5 \%$, Invesa, Barcelona, Spain) and meloxicam $(20 \mu \mathrm{g} / \mathrm{Kg} \mathrm{SC}$; Metacam, Boehringer Ingelheim España, Barcelona, Spain) for three days after surgery.

Three weeks after surgery, eight animals were randomly selected in each experimental group. The control group was treated with $5 \mathrm{~mL}$ of saline $(\mathrm{NaCl} 0.9 \%)$ and the treatment group with $1.5 \mathrm{mg} / \mathrm{Kg}$ of diacerein. Both treatments were administered daily and directly into the mouth using a $5 \mathrm{ml}$ syringe. The group distribution is represented in Fig. 1.

\section{Necropsy and preparation of histological specimens}

Rabbits were sacrificed by sodium pentobarbital overdose (100 mg/Kg IV; Dolethal, Vétoquinol, France) after sedation with ketamine $(25 \mathrm{mg} / \mathrm{Kg}$ IM; Imalgène 1000 , Merial, Toulouse, France). A complete necropsy of all the animals was performed to evaluate the presence of any alterations other than those in knee joints. After dissecting the articulation, two cylinders of $2.9 \mathrm{~mm}$ diameter and $8 \mathrm{~mm}$ length were obtained from the medial femoral condyle at the same anatomical locations of both knees of every animal and, within the same procedure a section of the articular capsule adjacent to the patellar ligament was obtained.

For evaluation, samples were divided in four groups (Fig. 1): CTRL: healthy non-operated knees of the placebotreated animals $(n=8)$; OA: operated knees of the placebo 


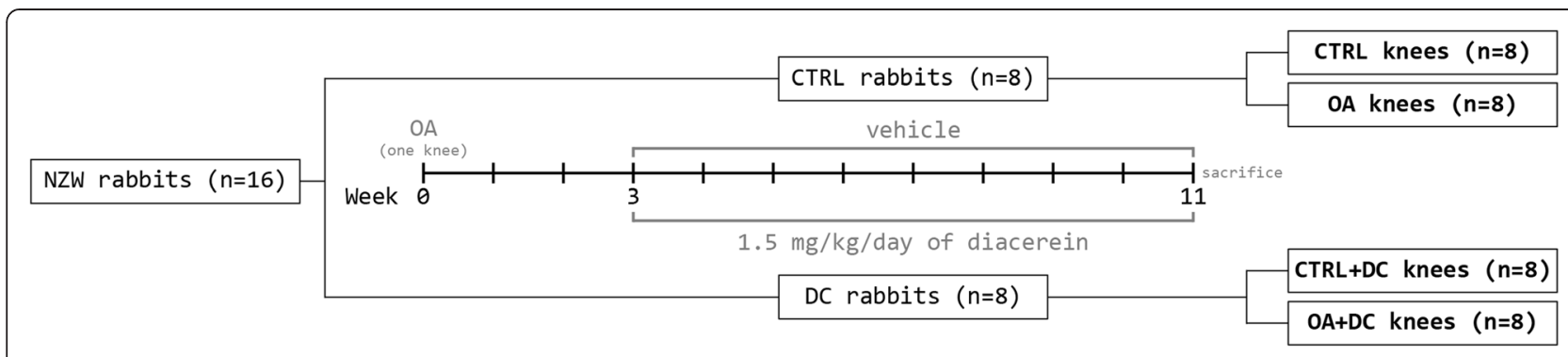

Figure 1 Experimental design. Groups of treatment

group $(n=8)$; CTRL + DC: non-operated healthy knees of the diacerein-treated animals $(n=8)$; OA $+\mathrm{DC}$ : osteoarthritic knees of the diacerein-treated animals $(n=8)$.

One trephine was decalcified with EDTA (ethylenediaminetetraacetic acid) in acid buffer (Osteodec, BioOptica, Milano, Italy) and, together with the articular capsule, was paraffin-embedded, sectioned and stained with haematoxylin-eosin (H-E) and safranin O-fast green. The synovial membrane slides were stained with $\mathrm{H}-\mathrm{E}$. The second trephine-cylinder was firstly used to evaluate the architecture of the cartilage and subchondral bone by micro-CT and later it was processed for undecalcified ground sections according to the method described by Donath (1985) [20], and stained using the Lévai-Laczkó method [21].

\section{Microscopic evaluation}

The gradation of the samples was performed in the same way as in a previously published article [19]

The synovial membrane and the decalcified bone and cartilage core biopsies were evaluated by two independent observers according to the already published guidelines [22,23]. The gradation of damage to the structures was as follows: in cartilage, chondrocyte and proteoglycan pathology from 0 (normal) to 4 (completely affected) and in tidemark and synovial membrane from 0 (normal) to 2 (osteoarthritic). In cartilage samples the parameters evaluated were: the cartilage pathology (cartilage surface characteristics), chondrocyte pathology (chondrocyte density and distribution, paying special attention to the clusters formation), proteoglycan pathology (degree of staining), tidemark integrity. In synovial membrane slides: lining cell characteristics (number of superficial cell layers), hyperplasia (presence or absence and its characteristics), and the presence or absence of cellular infiltrations.

Quantitative histology was performed using undecalcified sections applying previously published [24] morphological parameters by a masked examiner using PC-based image analysis programmes: Cell-sens 1.5 (Olympus Corporation, Japan) and Micro-image 4.0 (Media cybernetics, Bethesda, MD, USA). Firstly the reference lines were identified (Fig. 2) and after that, the programme automatically calculated the mean distances between these lines.

The evaluated parameters were [19]: Subchondral bone cortical thickness (SB.Th), total cartilage thickness (Cg.Th) and separately non-calcified (nCg.Th) and calcified cartilage thickness (cCg.Th) using the tidemark as reference (Fig. 2a), cartilage surface undulations (FI) (Fig. 2b), trabecular subchondral bone area (Tb.A) and trabecular separation (Tb.Sp). Tb.A and Tb.Sp were measured in a single subchondral bone region of interest (ROI) of $4 \times 2 \mathrm{~mm}$ beginning immediately after the subchondral cortical bone and including the entire width of the core biopsy except the margins (Fig. 2c). Tb.A was defined as the percentage of trabecular bone in this region and $\mathrm{Tb} . \mathrm{Sp}$ as the mean distance between trabeculae.

\section{Micro-computed tomography (micro-CT)}

Undecalcified sections were evaluated with a highresolution micro-CT (SkyScan 1172, Bruker micro CT NV, Kontich, Belgium). Two scans were performed for each sample, one to assess the microstructure of the subchondral bone and the other to visualise the articular cartilage using previously published parameters [19]. Images were reconstructed based on Feldkamp's convolution back-projection algorithm [25], and segmented into binary images. The following direct metric indices were measured: bone volumetric fraction (BV/TV), trabecular thickness (Tb.Th) and separation (Tb.Sp), and trabecular number (Tb.N) [26]. Directly assessed non-metric parameters were also calculated, including the trabecular bone pattern factor (Tb.Pf), which is an inverse measure of trabecular connectivity [27], the structural model index (SMI), which estimates the prevalence of rod-like or platelike trabeculae [28], and the degree of anisotropy (DA) describing the orientation of trabeculae [29]. Additionally, the volumetric bone mineral density (vBMD) was determined by direct calibration against attenuation coefficients of two hydroxyapatite phantoms $\left(250\right.$ and $\left.750 \mathrm{mg} / \mathrm{cm}^{3}\right)$. Regarding the cartilage, the average thickness of the noncalcified cartilage layer (nCg.Th) and its volume (nCg.V) were 3-D quantified. 


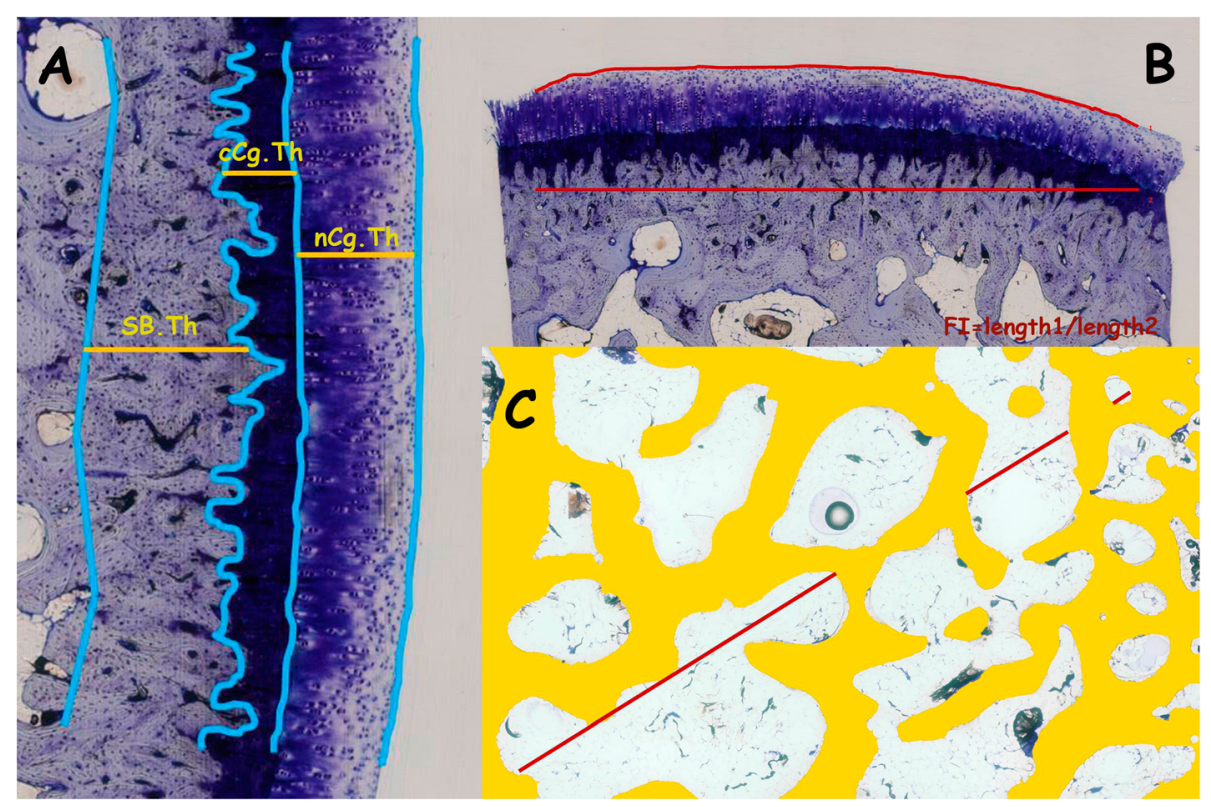

Figure 2 Representative images of the measurements made in undecalcified sections: a cartilage and subcondral bone cortical thickness (nCg.Th; cCg.Th; Cg.Th = nCg.Th + cCg.Th) SB.Th). b. Surface undulation (FI). c Trabecular subchondral bone measurements in a ROI. Tb.A: \% of trabecular bone in the ROI; Tb.Sp measured on the diagonal of ROI (Tb.Sp = (1/Tb.N)-Tb.Th)

\section{Statistical analysis}

The statistical analysis was performed with SPSS 19 for Windows (IBM, Armonk, NY, USA). The normality of the variables was checked by the Shapiro-Wilk test. The statistical comparison of normal variables was performed using ANOVA and the Levene's test was used to assess the equality of variances. Post-hoc analysis was carried out using the Tukey's HSD test for parameters with equal variances or the Games-Howell test for parameters with different variances. For non-normal variables, a statistical comparison was performed using the KruskalWallis $\mathrm{H}$ test and a post-hoc analysis using the Dunn's test. The statistical significance level was set at $p<0.05$ for all parameters.

\section{Results}

No changes in weight or general condition were observed during the experimental protocol. The only outstanding fact was that animals treated with diacerein presented urine discoloration; however, no pathological changes in the kidneys or the rest of the organs were detected in necropsies.

Out of the total of 32 harvested joints, except for one excluded due to infection, 31 presented an adequate status to be histologically analysed.

\section{Quantitative histological results}

Mean and standard deviation (SD) of the parameters quantified on undecalcified samples are shown in Table 1.
The subchondral bone parameters measured in these calcified slices obtained from CTRL and OA groups were similar and showed no changes in both treated groups $(\mathrm{CTRL}+\mathrm{DC}$ and $\mathrm{OA}+\mathrm{DC})$. Regarding the cartilagerelated parameters, non-calcified cartilage thickness (nCg.Th) showed a significant increase in the OA group compared to CTRL $(p=0.024)$ and CTRL + DC $(p=$ 0.009 ). The $\mathrm{OA}+\mathrm{DC}$ group (operated knee of the diacerein-treated animals) showed a decrease of the $\mathrm{nCg}$. Th with respect to the OA, reaching intermediate values halfway between CTRL/CTRL + DC and OA but showing no significant differences compared to none of them. For the total cartilage thickness (Cg.Th) differences between CTRL and OA groups are not significant, unlike between OA and CTRL + DC groups ( $p=$ $0.029)$. In this case, as well as in the non-calcified cartilage, the $\mathrm{OA}+\mathrm{DC}$ group showed intermediate values between controls and OA. In relation to the cartilage surface undulations (FI) there were no statistical differences between groups, but an almost significant reduction $(p=0.06)$ of fibrillation was found between OA and $\mathrm{OA}+\mathrm{DC}$ groups (Fig. 3), the latter reaching values similar to both control groups (CTRL and CTRL + DC).

In the microscopic images of the calcified samples stained with Levai-Lazckó (Fig. 3 A-D), despite small differences in the statistical analysis, several differences between groups were observed. Cartilage thickness (Cg.Th) of the OA groups (3B) was thicker than the others, the $\mathrm{OA}+\mathrm{DC}$ group (3D) reaching an intermediate thickness halfway between controls (3A and $3 \mathrm{C}$ ) and OA. Another 
Table 1 Quantitative histological results

\begin{tabular}{|c|c|c|c|c|}
\hline & $\begin{array}{l}\text { CTRL } \\
\text { mean } \pm \text { std.dev }\end{array}$ & $\begin{array}{l}\text { OA } \\
\text { mean } \pm \text { std.dev }\end{array}$ & $\begin{array}{l}C T R L+D C \\
\text { mean } \pm \text { std.dev }\end{array}$ & $\begin{array}{l}\mathrm{OA}+\mathrm{DC} \\
\text { mean } \pm \text { std.dev }\end{array}$ \\
\hline \multicolumn{5}{|c|}{ Subchondral bone } \\
\hline Tb.A $\left(\mathrm{mm}^{2}\right)$ & $46.37 \pm 5.41$ & $47.93 \pm 7.01$ & $50.69 \pm 7.34$ & $45.54 \pm 6.83$ \\
\hline Tb.Sp ( $\mu \mathrm{m})$ & $514.93 \pm 235.24$ & $437.79 \pm 211.86$ & $534.56 \pm 222.56$ & $590.16 \pm 272.66$ \\
\hline SB.Th $(\mu \mathrm{m})$ & $306.57 \pm 76.46$ & $251.60 \pm 67.43$ & $264.07 \pm 28.36$ & $268.20 \pm 47.01$ \\
\hline \multicolumn{5}{|l|}{ Cartilage } \\
\hline $\mathrm{Fl}(\mathrm{mm})$ & $1.08 \pm 0.07$ & $1.22 \pm 0.14$ & $1.10 \pm 0.16$ & $1.10 \pm 0.05$ \\
\hline Cg.Th $(\mu \mathrm{m})$ & $493.73 \pm 80.73$ & $620.12 \pm 114.82^{c}$ & $473.82 \pm 87.57^{b}$ & $571.12 \pm 96.73$ \\
\hline nCg.Th $(\mu m)$ & $338.64 \pm 65.03^{b}$ & $465.23 \pm 88.92^{a, c}$ & $322.19 \pm 82.98^{b}$ & $409.79 \pm 82.56$ \\
\hline cCg.Th $(\mu \mathrm{m})$ & $155.09 \pm 24.94$ & $167.75 \pm 43.01$ & $151.69 \pm 28.05$ & $161.33 \pm 42.09$ \\
\hline
\end{tabular}

Comparison of the histological parameters obtained from undecalcified samples between experimental groups. All variables were normally distributed, except Fl. Statistical significance $p<0.05:{ }^{\text {a }}$ vs. CTRL; ${ }^{\text {b }}$ vs. OA; ${ }^{c}$ vs. CTRL + DC; ${ }^{d}$ vs. OA + DC

important finding that may be visualised in the images is that the OA group had more superficial fibrillation than the others and the OA + DC surface is similar to those of the controls. The distribution of chondrocytes in the cartilage layer, although the cell distribution and cluster formation was not evaluated in these calcified samples, was different too, showing more disorganisation in the OA group (3B) than in the others. Finally, in the image of the OA group the loss of the part of the calcified cartilage (cCg.Th) could be observed, unlike in the others (3A, 3C, 3D).

\section{Qualitative histological results}

The values of microscopic grading for the decalcified cartilage samples and for the synovial membrane are shown in Table 2 and Fig. 4.
The parameters that assess changes in non-calcified cartilage showed no differences between the four groups, although the results of the severity of cartilage pathology of the $\mathrm{OA}+\mathrm{DC}$ group (osteoarthritic knees of the diacerein-treated animals) are halfway between both control groups (CTRL and CTRL + DC) and OA, without any statistical differences between them.

Two of the variables determined in the synovial membrane, that is, the features of the lining cells and the presence of hyperplasia, showed statistical significance between the OA and CTRL group $(p<0.05)$, but there was no presence of inflammatory infiltrates. Between the two diacerein groups $(C T R L+D C$ and $\mathrm{OA}+\mathrm{DC})$ the only statistical difference is the presence of hyperplasia. $\mathrm{OA}+\mathrm{DC}$ shows significant differences compared to OA in both lining cells characteristics and hyperplasia (Fig. 3)

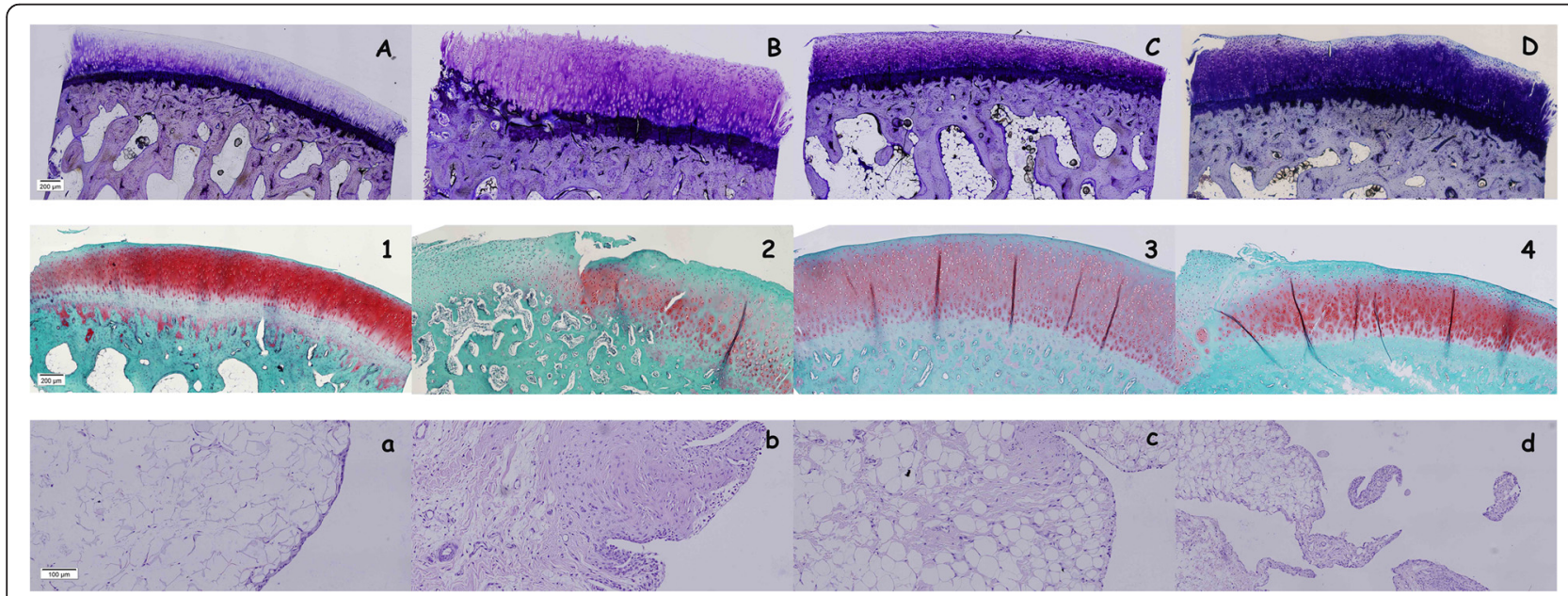

Figure 3 Representative histology images. A-D: Lévai-Laczkó stained undecalcified sections (A CTRL; B OA; C CTRL + DC; D OA + DC), magnification 10x. 1-4: safranin-O/fast green stained decalcified sections (1 CTRL; 2 OA; 3 CTRL + DC; 4 OA + DC), magnification 10x. a-d: synovial membrane sections stained with H-E (a CTRL; b OA; c CTRL + DC; d OA + DC), magnification 20x 
Table 2 Qualitative histological results

\begin{tabular}{|c|c|c|c|c|}
\hline & $\begin{array}{l}\text { CTRL } \\
\text { median }(25,75) \\
\mu \pm \text { std.dev }\end{array}$ & $\begin{array}{l}\text { OA } \\
\text { median }(25,75) \\
\mu \pm \text { std.dev }\end{array}$ & $\begin{array}{l}C T R L+D C \\
\text { median }(25,75) \\
\mu \pm \text { std.dev }\end{array}$ & $\begin{array}{l}\mathrm{OA}+\mathrm{DC} \\
\text { median }(25,75) \\
\mu \pm \text { std.dev }\end{array}$ \\
\hline \multicolumn{5}{|c|}{ Microscopic grading of cartilage alterations } \\
\hline \multirow[t]{2}{*}{ Severity of cartilage pathology } & $0(0,0.75)$ & $1(0.25,1.75)$ & $0(0,0.5)$ & $0(0,1)$ \\
\hline & $0.29 \pm 0.49$ & $1 \pm 0.82$ & $0.25 \pm 0.46$ & $0.5 \pm 0.76$ \\
\hline \multirow[t]{2}{*}{ Severity of chondrocyte pathology } & $0(0,1)$ & $1(0.25,2.5)$ & $1(0,1)$ & $1(1,3)$ \\
\hline & $0.57 \pm 0.79$ & $1.21 \pm 1.15$ & $0.56 \pm 0.5$ & $1.81 \pm 1.19$ \\
\hline \multirow[t]{2}{*}{ Severity of proteoglycan pathology } & $0(0,0)$ & $1(0,2)$ & $0(0,3)$ & $1.5(0,3)$ \\
\hline & $0.33 \pm 0.82$ & $1.29 \pm 1.5$ & $1.25 \pm 1.83$ & $1.56 \pm 1.68$ \\
\hline \multirow[t]{2}{*}{ Tidemark integrity } & $1(0.5,2)$ & $0(0,2)$ & $1(1,1)$ & $0.5(0,2)$ \\
\hline & $1.13 \pm 0.83$ & $0.67 \pm 1.03$ & $1 \pm 0.53$ & $0.88 \pm 0.99$ \\
\hline \multicolumn{5}{|c|}{ Microscopic grading of synovial changes } \\
\hline \multirow[t]{2}{*}{ Lining cells characteristics } & $0(0,0)^{b, c, d}$ & $1(1,1)^{a, c, d}$ & $0(0,1)^{a, b}$ & $0(0,0.75)^{a, b}$ \\
\hline & $0 \pm 0$ & $1 \pm 0.63$ & $0.4 \pm 0.55$ & $0.29 \pm 0.49$ \\
\hline \multirow[t]{2}{*}{ Presence of hyperplasia } & $0(0,0)^{b, c, d}$ & $1(1,2)^{a, c, d}$ & $0(0,0.25)^{a, b, d}$ & $1(0.25,1)^{a, b, c}$ \\
\hline & $0.13 \pm 0.35$ & $1.17 \pm 0.75$ & $0.2 \pm 0.45$ & $0.86 \pm 0.69$ \\
\hline \multirow[t]{2}{*}{ Cell infiltration characteristics } & $0(0,0)$ & $1(0,1)$ & $0(0,1)$ & $0(0,0.75)$ \\
\hline & $0 \pm 0$ & $0.67 \pm 0.52$ & $0.4 \pm 0.55$ & $0.29 \pm 0.49$ \\
\hline
\end{tabular}

Comparison between experimental groups with different scores for microscopic grading of cartilage and synovial alterations, obtained from decalcified samples. All parameters showed a non-normal distribution. Statistical significance $p<0.05:{ }^{a}$ vs. CTRL; ${ }^{b}$ vs. OA; ${ }^{c}$ vs. $C T R L+D C ;{ }^{d}$ vs. OA $+D C$

but not compared to the controls (CTRL and CTRL + $\mathrm{DC})$. The $\mathrm{OA}+\mathrm{DC}$ group reaches intermediate values halfaway between OA and CTRL for the three parameters evaluated in the synovial membrane.

As shown by the results of the histologic qualitative assessment of the decalcified samples, diacerein treatment resulted in less alteration of the cartilage surface but not in amelioration of the cellular alterations or in the loss of proteoglycan staining with respect to the osteoarthritic samples of the placebo-treated group (OA). Fig. $3.2(\mathrm{OA})$ and 3.4 (OA + DC) shows the chondrocyte disorganisation, with cells not arranged in rows, unlike in the controls (CTRL (3.1) and CTRL + DC (3.3)), and the evident formation of clusters, as well as the loss of the red stain, especially in the superficial layers of the cartilage.

In the synovial membrane of both control groups (Fig. 3.a and 3.c) the lining surface was thin, with one or two layers of cells, the surface was smooth, with no presence of short villi or finger-like hyperplasia, or of inflammatory infiltrations. The OA group (Fig. 3.b) was in the opposite situation, with a thick lining surface, profuse hyperplasia, presence of diffuse inflammatory infiltration and a deposition of fibrous tissue below the intima tissue. Finally, in the OA + DC group (Fig. 3.d), the number of superficial layers of cells tend to approach to the controls, as well as hyperplasia, these samples exhibiting less fibrous subintima tissue than the OA group.

\section{Micro-CT results}

The mean and SD values for the micro-CT parameters are shown in Table 3.

Comparing micro-CT data from the OA group versus the CTRL group, there were no differences in any of the microstructural parameters referring to the subchondral bone or volumetric bone mineral density (vBMD). In contrast, both the thickness of cartilage ( $\mathrm{nCg}$. Th) and its volume $(\mathrm{nCg} . \mathrm{V})$ are significantly increased in the OA group with respect to CTRL $(p=0.003$ and 0.018 , respectively) and to CTRL + DC $(p<0.001$ and $p=0.003)$ (Fig. 5).

For the $\mathrm{OA}+\mathrm{DC}$ group, the subchondral bone measurements show differences only with OA regarding the degree of anisotropy $(p=0.004)$, the rest of the results being similar to those obtained from the other groups. Nonetheless for cartilage parameters, OA + DC showed $\mathrm{nCg} . \mathrm{V}$ and $\mathrm{nCg}$.Th values close to those of the healthy control group (CTRL); thus, the value of $\mathrm{nCg}$. Th in the $\mathrm{OA}+\mathrm{DC}$ group presents statistically significant differences compared to OA $(p=0.041)$, whereas $\mathrm{nCg} . \mathrm{V}$ is almost significant $(p=0.053)$ (Fig. 5). 

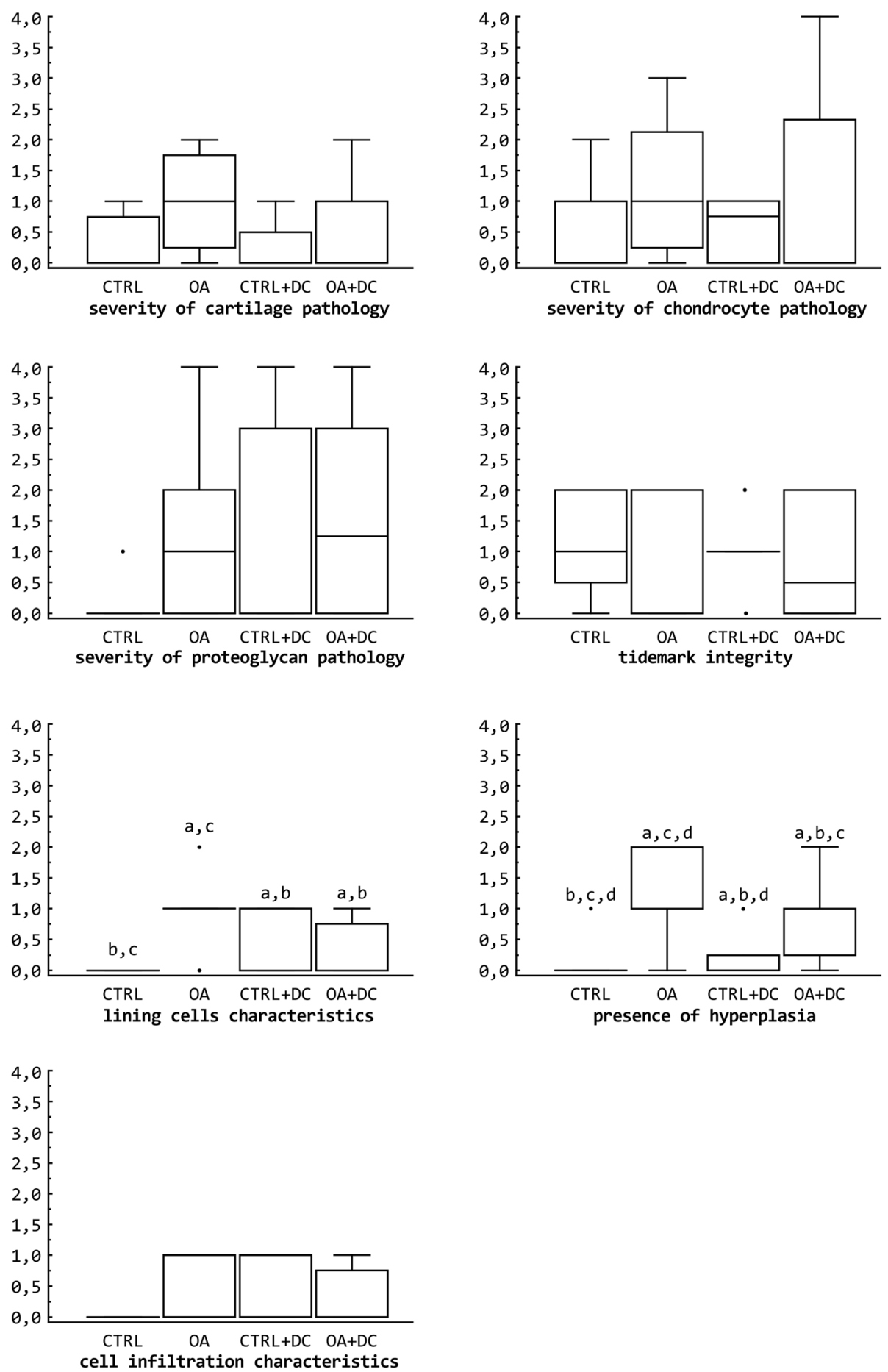

Figure 4 Box plot comparing the scores obtained by the different experimental groups for qualitative microscopic grading of cartilage and synovial alterations (decalcified samples). Outside values were defined as the values that were smaller than the lower quartile minus 1.5 times the interquartile range or larger than the upper quartile plus 1.5 times the interquartile range (inner fences). CTRL: healthy + placebo; OA: osteoarthritis + placebo; CTRL + DC: healthy + diacerein; OA + DC: osteoarthritis + diacerein. All parameters showed a non-normal distribution. Statistical significance $p<0.05$ : a vs. CTRL; b vs. OA; $c$ vs. CTRL + DC; $d$ vs. OA + DC 
Table 3 Micro-CT results

\begin{tabular}{|c|c|c|c|c|}
\hline & $\begin{array}{l}\text { CTRL } \\
\text { mean } \pm \text { std.dev }\end{array}$ & $\begin{array}{l}\text { OA } \\
\text { mean } \pm \text { std.dev }\end{array}$ & $\begin{array}{l}C T R L+D C \\
\text { mean } \pm \text { std.dev }\end{array}$ & $\begin{array}{l}\mathrm{OA}+\mathrm{DC} \\
\text { mean } \pm \text { std.dev }\end{array}$ \\
\hline \multicolumn{5}{|c|}{ Subchondral bone microstructure } \\
\hline BV/TV (\%) & $48.46 \pm 7.74^{c}$ & $49.37 \pm 4.38$ & $56.34 \pm 2.96^{\mathrm{a}}$ & $51.92 \pm 4.99$ \\
\hline Tb.Th $(\mu \mathrm{m})$ & $265.21 \pm 32.62$ & $288.75 \pm 60.43$ & $283.20 \pm 34.22$ & $281.18 \pm 56.18$ \\
\hline Tb.Sp $(\mu \mathrm{m})$ & $467.24 \pm 122.46^{c}$ & $423.12 \pm 66.21$ & $354.74 \pm 36.25^{a}$ & $433.27 \pm 54.03$ \\
\hline Tb.N $\left(\mathrm{mm}^{-1}\right)$ & $1.83 \pm 0.20$ & $1.76 \pm 0.29$ & $2.01 \pm 0.23$ & $1.89 \pm 0.30$ \\
\hline Tb.Pf $\left(\mathrm{mm}^{-1}\right)$ & $-3.27 \pm 1.49$ & $-3.24 \pm 0.51$ & $-4.16 \pm 1.54$ & $-4.01 \pm 1.15$ \\
\hline SMl & $-0.66 \pm 0.47$ & $-0.72 \pm 0.39$ & $-1.07 \pm 0.48$ & $-0.96 \pm 0.60$ \\
\hline DA & $0.71 \pm 0.06^{c, d}$ & $0.69 \pm 0.06^{c, d}$ & $0.57 \pm 0.09^{\mathrm{a}, \mathrm{b}}$ & $0.57 \pm 0.06^{\mathrm{a}, \mathrm{b}}$ \\
\hline \multicolumn{5}{|c|}{ Volumetric subchondral bone mineral density } \\
\hline $\operatorname{VBMD}\left(\mathrm{mg} / \mathrm{cm}^{3}\right)$ & $505.90 \pm 98.53$ & $512.78 \pm 52.52^{c}$ & $608.36 \pm 24.43^{b}$ & $548.69 \pm 58.95$ \\
\hline \multicolumn{5}{|c|}{ Cartilage morphology } \\
\hline nCg.Th $(\mu \mathrm{m})$ & $309.06 \pm 62.99^{b}$ & $494.80 \pm 169.59^{a, c, d}$ & $269.01 \pm 39.93^{b}$ & $359.75 \pm 48.62^{b}$ \\
\hline $\mathrm{nCg.V}\left(\mathrm{mm}^{3}\right)$ & $3.37 \pm 0.78^{b}$ & $5.69 \pm 2.72^{\mathrm{a}, \mathrm{c}}$ & $2.81 \pm 0.52^{b}$ & $3.71 \pm 0.50$ \\
\hline
\end{tabular}

Mean values \pm standard deviation of the micro-CT parameters obtained for all experimental groups. All variables showed a normal distribution, and only vBMD showed no homogeneity of variances. Statistical significance $p<0.05:{ }^{a}$ vs. CTRL; ${ }^{b}$ vs. OA; ${ }^{c}$ vs. CTRL $+D C ;{ }^{d}$ vs. OA + DC

In the healthy group treated with diacerein (CTRL + DC), the drug showed an anabolic effect on the subchondral bone structure, with an increase in bone volume fraction $(\mathrm{BV} / \mathrm{TV})$ versus CTRL $(p=0.029)$, besides a decrease in trabecular separation and degree of anisotropy ( $p=0.032$ and 0.001 respectively). The microstructural parameters measured in cartilage showed no difference between both control groups.

\section{Discussion}

Diacerein has been subject of study regarding its use in the treatment of human OA for some time now. Animal models have been widely used to study the efficacy of therapies in order to improve, fight or prevent OA. Surgically-induced OA models, resulting in joint instability, produce a gradual progression of the joint degeneration and mimic the pathogenesis and pathology of the human traumatic OA [30,31]. Since the first model carried out by Paatsama (1952) [32], many studies have surgically induced OA using meniscectomy and/or transection of the collateral and cruciate ligaments in different animal species [33]. Partial medial meniscectomy in rabbits results in mild to moderate changes in the joint, resembling those in humans, thus it has been extensively used for testing potential chondroprotective agents [34]. Our animal model was a combination of meniscectomy and ligament transection to avoid the great capacity of rabbits to heal the transected meniscus with fibrous tissue and, similarly to the results obtained by other authors, the results in the present study have shown that this model produced degenerative changes with respect to the healthy contralateral joint [30]. Although the animals were sacrificed at 11 weeks post-surgery, OA seemed to have begun developing, as proven by the swelling of the cartilage. The increase in volume and thickness of cartilage of the OA samples is due to a swelling phenomenon characteristic to the early stages of the disease. Several studies on animals of early OA

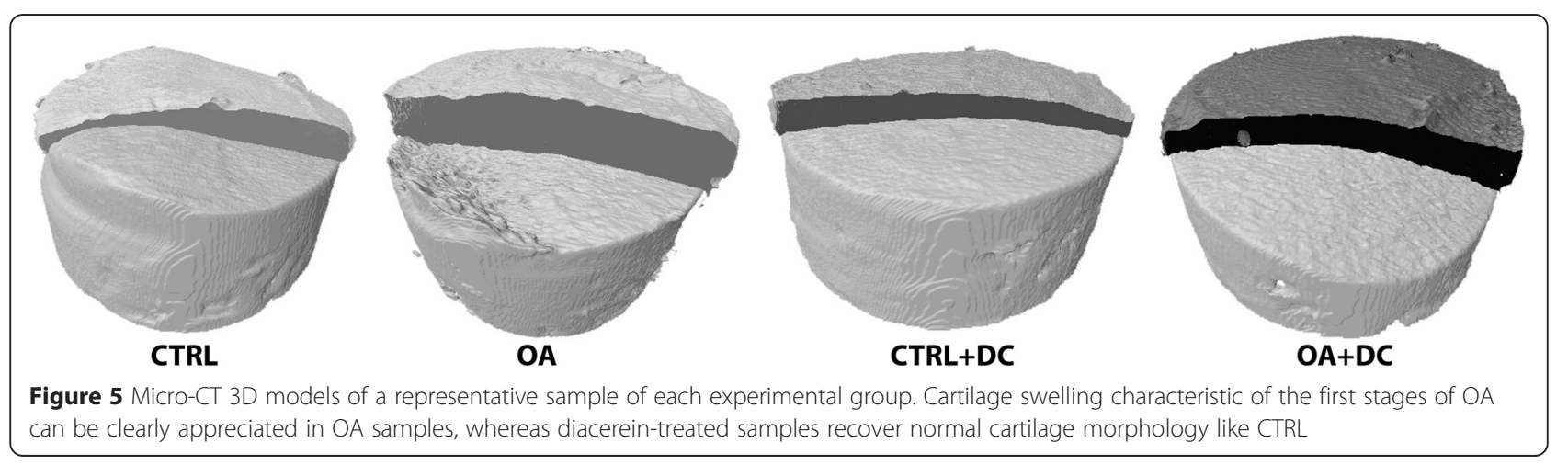


with magnetic resonance imaging and histomorphometry identified an initial phase of cartilage hypertrophy prior to its degeneration and loss $[35,36]$. The explanation has not been found yet, and it is not known whether it is the tissue expression to an inflammatory and repair phenomenon or if it represents a permanent damage of the joint tissue.

Special attention should be paid to the methodology used in this study. Samples of subchondral bone and articular cartilage were analysed using different quantitative and qualitative microscopic methods and micro-CT. Histologic assessment of OA is currently considered as the gold-standard for determining the presence, extent and severity of the disease using Mankin [37] or modified Mankin scoring systems, but recently the histomorphometry using computer analysis systems [24] has been introduced in $\mathrm{OA}$ studies with a greater degree of objectivity and reproducibility compared to previous qualitative studies.

Micro-CT has become in recent years the goldstandard for three-dimensional analysis of bone microstructure and its objectivity and reproducibility was comparable to histomorphometry. Although normally when the cartilage structure was quantified using micro-CT, the process involved complex staining techniques with radiopaque agents [38-40], in the present study the scan conditions have been adjusted to achieve a correct cartilage visualisation without contrast agents and it was enough to be able to quantify its morphology.

Our results revealed the potential anti-inflammatory effects of diacerein on osteoarthritis in two different ways: an improvement in the synovial membrane and a reduction in the thickness of the non-calcified cartilage. Previous studies revealed the beneficial effect of this drug not only for cartilage protection, but also for synovitis and reduction in bone erosion in a mice model of inflammatory arthritis [41]. The mechanisms of the antiinflammatory effect of diacerein have not yet been completely clarified but Tamura et al. (2002) [42] demonstrated, using inflammatory animal models, a different spectrum of anti-inflammatory activity of diacerein than those of NSAIDs, which may be due to the inhibition of IL-1 and the production of reactive oxygen species [42, 43]. The histologic evaluation of the synovial membrane in the OA group revealed moderate to severe inflammatory changes with thickening of the lining cell layer, hyperplasia and infiltration with inflammatory cells. In the diacerein-treated group $(\mathrm{OA}+\mathrm{DC})$ these changes are milder than in the OA group, although, as in a canine cruciate-deficiency model [12] the results are not statistically significant, as the animals of this $\mathrm{OA}+\mathrm{DC}$ group obtained values halfway between healthy and osteoarthritic ones. The same results were observed in the cartilage, where a reduction of the thickness is observed in treated animals with respect to the OA group.

In the paraffin-embedded samples there were no differences between groups (although the cartilage pathology is almost significant). A possible explanation could be the substantial variability due to the use of a qualitative subjective scoring system for their evaluation; another explanation (comparing these results with the calcified samples) could be that due to decalcification the integrity of the samples (chiefly bone and calcified cartilage) may have been altered [24]. However, in decalcified samples, the results for the OA + DC group were closer to normality than those obtained for the OA group.

In the samples evaluated by a histomorphometric quantitative methodology, results showed improvement in the diacerein-treated group compared to the one treated with placebo, although differences were not significant. In cartilage parameters there were statistical differences in $\mathrm{Cg}$.Th and $\mathrm{nCg}$.Th between the $\mathrm{OA}$ group and the CTRL + DC group and for the latter also with the CTRL group. There were no differences between $\mathrm{OA}$ and $\mathrm{OA}+\mathrm{DC}$, but nor between $\mathrm{OA}+\mathrm{DC}$ and CTRL + DC and the tendency of values was to approximate joint values to normal. Regarding FI -an important parameter because it makes a clear distinction between ill and healthy animals [44]- there were no statistical differences between groups, but there is a tendency of approximating values of $\mathrm{OA}+\mathrm{DC}$ to those of controls.

The results obtained from the undecalcified samples were better than those from the decalcified ones because of a better conservation of the tissue structure and because the computer evaluation has a greater degree of objectivity, accuracy and reproducibility, as well as the micro-CT [24], with comparable results between both. In our study, the subchondral bone showed no statistical differences, so further studies may be needed in this regard.

Micro-CT data have shown an anabolic effect of diacerein on subchondral trabecular microstructure in both healthy and osteoarthritic samples (though less pronounced in the latter). At the same time, the treatment has been able to stop the swelling of cartilage in OA samples, recovering $\mathrm{nCg}$.Th and $\mathrm{nCg}$. $\mathrm{V}$ values very close to the CTRL group.

The results of the present animal study conducted in rabbits suggest the possible efficacy of diacerein to slow the progression of the disease in an early model of OA, as shown in previous studies in vitro [45], in different animal models $[12,42,45,46]$ and in clinical trials [7, $16,47]$; although the comparison of different studies is difficult because of their high degree of heterogeneity (different models conducted in different animal species and humans, different time schedule and others). To 
complete this study and correctly evaluate the obtained results, it may be necessary to evaluate the effects of diacerein treatment in the same animal model with a more advanced OA.

\section{Conclusions}

Oral diacerein as OA treatment has been able to reduce cartilage swelling, surface alterations and the inflammation of the synovial membrane in the early stages of OA in a rabbit surgical model. Regarding the subchondral bone, diacerein has shown in healthy bone, but not in the osteoarthritic one, the ability to increase bone volume and mineral density, indicating a surprising effect of this drug on the bone structure, whose results have not been published up to now.

The study also demonstrates the validity of the animal model, and that micro-CT could be a valid technique to detect morphological changes in articular cartilage with comparable results to histomorphometry.

Further studies on long-term OA animal models and well-conducted clinical trials may be required to confirm or exclude the efficacy of diacerein in the treatment of knee OA.

\section{Abbreviations \\ OA: Osteoarthritis; SYSADOAs: symptomatic slow-acting drugs for osteoarthritis; NSAIDS: non-steroidal anti-inflammatory drugs; ARRIVE: Animal Research: Reporting in vivo experiments; ACLT: anterior cruciate ligament transection; SO: safranin-O staining; H-E: Hematoxylin-eosin.}

\section{Competing interests}

The authors declare that they have no competing interests.

\section{Authors' contributions}

$\mathrm{DG}, J \mathrm{RC}, \mathrm{FM}$ and AGC participated in the conception and design of the study. The animal model and the histological analyses were performed by MP, ML, FM and AGC; micro-CT assessments were conducted by DG and JRC. All authors have collaborated on data analysis, interpretation of results, drafting and revision of article for final approval.

\section{Acknowledgements}

The authors gratefully acknowledge the assistance of Natalia Miño, Mariano López and Oscar Varela of the Department of Veterinary Clinical Sciences, University of Santiago de Compostela, for their contribution in surgery procedures and drug administration, of the staff at the Animal Research Facility, University of Santiago de Compostela, for taking care of the animals and Bioiberica (Barcelona, Spain), which provided drugs for the study. The authors are grateful to the Directorate-General of Research, Development and Innovation, Ministry of Economy and Industry, Xunta de Galicia for funding this work through research project 09CSA008E, co-financed by the European Regional and Social Funds (FEDER and FSE) and by a grant from the Fundación Salud 2000. The funders have no role in the study design, data analysis and interpretation, writing of the manuscript or decision to submit it for publication.
\end{abstract}

\footnotetext{
Author details

'Veterinary Clinical Sciences, University of Santiago de Compostela (USC), Campus Universitario, s/n, 27002, Lugo, Spain. ${ }^{2}$ Trabeculae S.L., Parque Tecnolóxico de Galicia, 32900 San Cibrao das Viñas, Ourense, Spain. ${ }^{3}$ Orthopedic Surgery Service, USC University Hospital Complex, Travesía de Choupana, s/n, 15706, Santiago de Compostela, Spain.
}

Received: 12 September 2014 Accepted: 22 June 2015

Published online: 02 July 2015

\section{References}

1. Martel-Pelletier J. Pathophysiology of osteoarthritis. Osteoarthritis Cartilage. 2004;12:S31-33.

2. Martel-Pelletier J, Pelletier JP. Is osteoarthritis a disease involving only cartilage or other articular tissues? Eklem Hastalik Cerrahisi. 2010;21:2-14.

3. Huskisson EC, Berry H, Gishen P, Jubb RW, Whitehead J. Effects of antiinflammatory drugs on the progression of osteoarthritis of the knee. LINK Study Group. Longitudinal Investigation of Nonsteroidal Antiinflammatory Drugs in Knee Osteoarthritis. J Rheumatol. 1995;22:1941-6.

4. Davies NM, Røseth AG, Appleyard CB, McKnight W, Del Soldato P, Calignano A, et al. NO-naproxen vs. naproxen: ulcerogenic, analgesic and antiinflammatory effects. Aliment Pharmacol Ther. 1997;11:69-79.

5. Qvist P, Bay-Jensen AC, Christiansen C, Dam EB, Pastoureau P, Karsdal MA. The disease modifying osteoarthritis drug (DMOAD): Is it in the horizon? Pharmacol Res. 2008;58:1-7.

6. Fernandes JC, Martel-Pelletier J, Pelletier JP. The role of cytokines in osteoarthritis pathophysiology. Biorheology. 2002;39:237-46.

7. Pelletier JP, Caron JP, Evans C, Robbins PD, Georgescu HI, Jovanovic D, et al. In vivo suppression of early experimental osteoarthritis by interleukin-1 receptor antagonist using gene therapy. Arthritis Rheum. 1997;40:1012-9.

8. Martel-Pelletier J, Mineau F, Jolicoeur FC, Cloutier JM, Pelletier JP. In vitro effects of diacerhein and rhein on interleukin 1 and tumor necrosis factoralpha systems in human osteoarthritic synovium and chondrocytes. J Rheumatol. 1998;25:753-62.

9. Yaron M, Shirazi I, Yaron I. Anti-interleukin-1 effects of diacerein and rhein in human osteoarthritic synovial tissue and cartilage cultures. Osteoarthritis Cartilage. 1999;7:272-80.

10. Felisaz N, Boumediene K, Ghayor C, Herrouin JF, Bogdanowicz P, Galerra P, et al. Stimulating effect of diacerein on TGF-beta1 and beta2 expression in articular chondrocytes cultured with and without interleukin-1. Osteoarthritis Cartilage. 1999;7:255-64.

11. Fidelix TS, Soares BG, Trevisani VF. Diacerein for osteoarthritis. Cochrane Database Syst Rev. 2006;25, CD005117.

12. Smith Jr GN, Myers SL, Brandt KD, Mickler EA, Albrecht ME. Diacerhein treatment reduces the severity of osteoarthritis in the canine cruciatedeficiency model of osteoarthritis. Arthritis Rheum. 1999;42:545-54.

13. Rezende MU, Gurgel HM, Vilaça Junior PR, Kuroba RK, Lopes AS, Phillipi RZ, et al. Diacerhein versus glucosamine in a rat model of osteoarthritis. Clinics (Sao Paulo). 2006;61:461-6.

14. Rintelen B, Neumann K, Leeb BF. A meta-analysis of controlled clinical studies with diacerein in the treatment of osteoarthritis. Arch Intern Med 2006;166:1899-906

15. Bartels EM, Bliddal H, Schondorff PK, Altman RD, Zhang W, Christensen R. Symptomatic efficacy and safety of dicerein in the treatment of osteoarthritis: a meta-analysis of randomized placebo-controlled trials. Osteoarthritis Cartilage. 2010;18:289-96.

16. Pavelka K, Trc T, Karpas K, Vitek $P$, Sedlácková M, Vlasáková V, et al. The efficacy and safety of diacerein in the treatment of painful osteoarthritis of the knee: a randomized, multicenter, double-blind, placebo-controlled study with primary end points at two months after the end of a three-month treatment period. Arthritis Rheum. 2007;56:4055-64.

17. Singh K, Sharma R, Rai J. Diacerein as adjuvant to diclofenac sodium in osteoarthritis knee. Int J Rheum Dis. 2012;15:69-77.

18. Kilkenny C, Browne WJ, Cuthill IC, Emerson M, Altman DG. Improving Bioscience Research Reporting: The ARRIVE Guidelines for Reporting Animal Research. Osteoarthritis Cartilage. 2012;20:256-60.

19. Permuy M, Guede D, López-Peña M, Muñoz F, González-Cantalapiedra A, Caeiro JR. Effects of glucosamine and risedronate alone or in combination in an experimental rabbit model of osteoarthritis. BMC Vet Res. 2014;10:97.

20. Donath $\mathrm{K}$. The diagnostic value of the new method for the study of undecalcified bones and teeth with attached soft tissue (Säge-Schliff (sawing and grinding) technique). Pathol Res Pract. 1985;179:631-3.

21. Laczkó J, Lévai G. A simple differential staining method for semi-thin sections of ossifying cartilage and bone tissues embedded in epoxy resin. Mikroskopie. 1975;31:1-4.

22. Cook JL, Kuroki K, Visco D, Pelletier JP, Schulz L, Lafeber FP. The OARSI histopathology initiative - recommendations for histological assessments of osteoarthritis in the dog. Osteoarthritis Cartilage. 2010;18:S66-79.

23. Laverty S, Girard CA, Williams JM, Hunziker EB, Pritzker KP. The OARSI histopathology initiative - recommendations for histological assessments of osteoarthritis in the rabbit. Osteoarthritis Cartilage. 2010;18:S53-65. 
24. Pastoureau PC, Hunziker EB, Pelletier JP. Cartilage, bone and synovial histomorphometry in animal models of osteoarthritis. Osteoarthritis Cartilage. 2010;18:S106-112

25. Feldkamp LA, Davis LC, Kress JW. Practical cone-beam algorithm. J Opt Socof Am A. 1984;1:612-9.

26. Hahn M, Vogel M, Pompesius-Kempa M, Delling G. Trabecular bone pattern factor - a new parameter for simple quantification of bone microarchitecture. Bone 1992:13:327-30

27. Hildebrand T, Laib A, Müller R, Dequeker J, Rüegsegger P. Direct threedimensional morphometric analysis of human cancellous bone: microstructura data from spine, femur, iliac crest, and calcaneus. J Bone Miner Res. 1999:14:1167-74.

28. Hildebrand T, Rüegsegger P. Quantification of bone microarchitecture with the structure model index. Comput Methods Biomech Biomed Eng. 1997;1:15-23.

29. Odgaard A, Jensen EB, Gundersen HJ. Estimation of structural anisotropy based on volume orientation. A new concept. J Microsc. 1990;157:149-62.

30. Bendele AM. Animal models of osteoarthritis. J Musculoskelet Neuronal Interact. 2001;1:363-76.

31. Pritzker KP. Animal models for osteoarthritis: processes, problems and prospects. Ann Rheum Dis. 1994:53:406-20.

32. Paatsama S. Ligament injuries of the canine stifle joint: A clinical and experimental study, Master's thesis. Helsinki: Veterinary College; 1952.

33. Kamekura S, Hoshi K, Shimoaka T, Chung U, Chikuda H, Yamada T, et al. Osteoarthritis development in novel experimental mouse models induced by knee joint instability. Osteoarthritis Cartilage. 2005;13:632-41.

34. Moskowitz RW, Davis W, Sammarco J, Martens M, Baker J, Mayor M, et al. Experimentally induced degenerative joint lesions following partial meniscectomy in the rabbit. Arthritis Rheum. 1973;16:397-405.

35. Calvo E, Palacios I, Delgado E, Ruiz-Cabello J, Hernández P, Sánchez-Pernaute $\mathrm{O}$, et al. High-resolution MRI detects cartilage swelling at the early stages of experimental osteoarthritis. Osteoarthritis Cartilage. 2001;9:463-72.

36. Calvo E, Palacios I, Delgado E, Sánchez-Pernaute O, Largo R, Egido J, et al. Histopathological correlation of cartilage swelling detected by magnetic resonance imaging in early experimental osteoarthritis. Osteoarthritis Cartilage. 2004;12:878-86.

37. Mankin HJ, Dorfman H, Lippiello L, Zarins A. Biochemical and metabolic abnormalities in articular cartilage from osteo-arthritic human hips. II. Correlation of morphology with biochemical and metabolic data. J Bone Joint Surg Am. 1971:53:523-37.

38. Palmer AW, Guldberg RE, Levenston ME. Analysis of cartilage matrix fixed charge density and three-dimensional morphology via contrast-enhanced microcomputed tomography. Proc Natl Acad Sci USA. 2006;103:19255-60.

39. Xie L, Lin AS, Levenston ME, Guldberg RE. Quantitative assessment of articular cartilage morphology via EPIC-microCT. Osteoarthritis Cartilage. 2009:17:313-20.

40. Yoo WJ, Cheon JE, Lee HR, Cho TJ, Choi IH. Physeal growth arrest by excessive compression: histological, biochemical, and micro-CT observations in rabbits. Clin Orthop Surg. 2011;3:309-14.

41. Douni E, Sfikakis PP, Haralambous S, Fernandes P, Kollias G. Attenuation of inflammatory polyarthritis in TNF transgenic mice by diacerein: comparative analysis with dexamethasone, methotrexate and anti-TNF protocols. Arthritis Res Ther. 2004;6:R65-72.

42. Tamura T, Shirai T, Kosaka N, Ohmori K, Takafumi N. Pharmacological studies of diacerein in animal models of inflammation, arthritis and bone resorption. Eur J Pharmacol. 2002;44:81-7.

43. Tamura $T$, Kosaka N, Ishiwa J, Sato T, Nagase H, Ito A. Rhein, an active metabolite of diacerein, down-regulates the production of pro-matrix metalloproteinases-1, $-3,-9$ and -13 and up-regulates the production of tissue inhibitor of metalloproteinase-1 in cultured rabbit articular chondrocytes. Osteoarthritis Cartilage. 2001;9:257-63.

44. Pastoureau P, Leduc S, Chomel A, De Ceunink F. Quantitative assessment of articular cartilage and subchondral bone histology in the meniscectomized guinea pig model of osteoarthritis. Osteoarthritis Cartilage. 2003;11:412-23.

45. Brandt KD, Smith G, Kang SY, Myers S, O'Connor B, Albretch M. Effects of diacerhein in an accelerated canine model of osteoarthritis. Osteoarthritis Cartilage. 1997:5:438-49.
46. Hwa SY, Burkhardt D, Little C, Ghosh P. The effects of orally administered diacerein on cartilage and subchondral bone in an ovine model of osteoarthritis. J Rheumatol. 2001;28:825-34.

47. Brahmachari B, Chatterjee S, Ghosh A. Efficacy and safety of diacerein in early knee osteoarthritis: a randomized placebo-controlled trial. Clin Rheumatol. 2009;28:1193-8

\section{Submit your next manuscript to BioMed Central and take full advantage of:}

- Convenient online submission

- Thorough peer review

- No space constraints or color figure charges

- Immediate publication on acceptance

- Inclusion in PubMed, CAS, Scopus and Google Scholar

- Research which is freely available for redistribution 